This is the accepted version of the following article:

Huertas C.S., Aviñó A., Kurachi C., Piqué A., Sandoval J., Eritja R., Esteller M., Lechuga L.M.. Label-free DNA-methylation detection by direct ds-DNA fragment screening using poly-purine hairpins. Biosensors and Bioelectronics, (2018). 120. : 47 - .

10.1016/j.bios.2018.08.027,

which has been published in final form at https://dx.doi.org/10.1016/j.bios.2018.08.027 (c) https://dx.doi.org/10.1016/j.bios.2018.08.027. This manuscript version is made available under the CC-BY-NC-ND 4.0 license http://creativecommons.org/licenses/by-nc-nd/4.0/ 


\section{Author's Accepted Manuscript}

Label-free DNA-methylation detection by direct dsDNA fragment screening using poly-purine hairpins

César S. Huertas, Anna Aviñó, Cristina Kurachi, Albert Piqué, Juan Sandoval, Ramón Eritja, Manel Esteller, Laura M. Lechuga

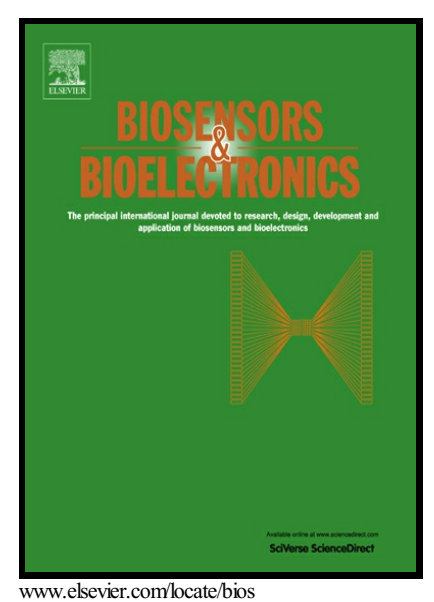

PII: $\quad$ S0956-5663(18)30623-7

DOI: $\quad$ https://doi.org/10.1016/j.bios.2018.08.027

Reference: BIOS10691

To appear in: Biosensors and Bioelectronic

Received date: 17 May 2018

Revised date: 25 July 2018

Accepted date: 11 August 2018

Cite this article as: César S. Huertas, Anna Aviñó, Cristina Kurachi, Albert Piqué, Juan Sandoval, Ramón Eritja, Manel Esteller and Laura M. Lechuga, Label-free DNA-methylation detection by direct ds-DNA fragment screening using poly-purine hairpins, Biosensors and Bioelectronic, https://doi.org/10.1016/j.bios.2018.08.027

This is a PDF file of an unedited manuscript that has been accepted for publication. As a service to our customers we are providing this early version of the manuscript. The manuscript will undergo copyediting, typesetting, and review of the resulting galley proof before it is published in its final citable form. Please note that during the production process errors may be discovered which could affect the content, and all legal disclaimers that apply to the journal pertain. 


\title{
Label-free DNA-methylation detection by direct ds-DNA fragment screening using poly-purine hairpins
}

César S. Huertas, ${ }^{1,2, a}$ Anna Aviñó, ${ }^{2,3}$ Cristina Kurachi, ${ }^{1,2}$ Albert Piqué, ${ }^{1,2}$ Juan Sandoval, ${ }^{4, b}$ Ramón Eritja, ${ }^{2,3}$ Manel Esteller, ${ }^{4,5,6}$ Laura M. Lechuga ${ }^{1,2, *}$

${ }^{1}$ Nanobiosensors and Bioanalytical Applications Group, Catalan Institute of Nanoscience and Nanotechnology (ICN2), CSIC and BIST, Campus UAB, Bellaterra, 08193 Barcelona, Spain

${ }^{2}$ CIBER-BBN Networking Center on Bioengineering, Biomaterials and Nanomedicine.

${ }^{3}$ Department of Chemical and Biomolecular Nanotechnology, Institute for Advanced Chemistry of Catalonia (IQAC), CSIC, c/Jordi Girona 18-26, 08034 Barcelona, Spain.

${ }^{4}$ Cancer Epigenetics and Biology Program, Bellvitge Biomedical Research Institute, L'Hospitalet, Barcelona, Catalonia, Spain.

${ }^{5}$ Physiological Sciences Department, School of Medicine and Health Sciences, University of Barcelona (UB), Catalonia, Spain.

${ }^{6}$ Institució Catalana de Recerca i Estudis Avançats (ICREA), Barcelona, Catalonia, Spain

Present Addresses

${ }^{a}$ School of Engineering, RMIT University, Melbourne (Australia).

${ }^{\mathrm{b} B i o m a r k e r s ~ a n d ~ P r e c i s i o ́ n ~ M e d i c i n e ~ U n i t, ~ E p i g e n o m i c s ~ c o r e ~ f a c i l i t y, ~ H e a l t h ~ R e s e a r c h ~ I n s t i t u t e ~ L a ~ F e, ~ A v e n i d a ~}$ Fernando Abril Martorell 106, 46026 Valencia (Spain).

*corresponding author: laura.lechuga @icn2.cat

\begin{abstract}
Cancer diagnosis continuously evolves due to the better understanding of tumorigenic processes. DNAmethylation is consolidated as an effective biomarker for cancer prognosis and diagnostic even in tumours of unknown origin. The reversibility of this epigenetic mechanism also places it as a high-profile tool for the development of more sophisticated and personalized therapies. Current methodologies, such as bisulfite conversion or PCR amplification, rely on complex procedures that make difficult the standardization of epigenetics analyses. Here we present an optical biosensor methodology based on Surface Plasmon Resonance that employs poly-purine reverse-Hoogsten hairpin probes capable of interacting directly with ds-DNA fragments by triple helix formation. The direct interaction with the material of interest can greatly enhance the reliability of the analysis providing a more accurate and precise diagnosis. We have demonstrated the capabilities of our methodology for the direct capture of ds-DNA fragments and specific methyl-cytosine quantification. Our poly-purine hairpin probe demonstrated the specific capture of dsDNA fragments while the standard duplex-forming probes failed to do so.. In addition, the biosensor methodology showed a strong correlation with the different DNA methylation status between the sequences with a low signal variation $(\leq 8 \% \mathrm{CV})$ along 35 hybridization/regeneration cycles. Through its
\end{abstract}


straightforward procedure and versatility of detecting different DNA modifications related to the DNA methylation process, we anticipate that our strategy will enable a greater level of accuracy and precision in cancer diagnostics making a strong impact on the development of personalized therapies.

GRAPHICAL ABSTRACT

KEYWORDS: DNA methylation, DNA capture, Surface Plasmon Resonance, Biosensor, Diagnosis, Triplex

\section{INTRODUCTION}

One of the main objectives in the improvement of cancer management is the finding of highly selective, targeted therapies. Cancers are heterogeneous not only in terms of histology and clinical outcome, but also at the molecular level (Ellsworth et al., 2017; Sartore-Bianchi et al., 2016). Therefore, each cancer also differs in its response to therapeutic interventions depending on both, the cancer origin and the patient. The most common predictive biomarkers for cancer diagnosis are mainly proteins, produced as a consequence of the tumorigenic process (Sölétormos et al., 2016). Their presence in tissues or blood can be related to cancer, but little information can be inferred about which cellular mechanism has been altered. Identifying the mechanisms involved in the cancer will not only provide information of how to restore the alteration but also to foresee the tumorigenic process.

Epigenetics plays a key role in cancer origin and great efforts have been made in order to decipher the exerted influence of these inherited marks in tumors (Heard and Martienssen, 2014). One of the most relevant epigenetic mechanisms is the methylation of DNA, which was the first epigenetic mark associated with cancer as a consequence of the alteration in normal gene regulation (Feinberg and Vogelstein, 1983). Since then it has been regarded as a promising biomarker for cancer prevention due to its reversibility and potential impact in the development of personalized medicine (Jankowska et al., 2015).

DNA methylation consists of the addition of a methyl group $\left(-\mathrm{CH}_{3}\right)$ at the C-5 position of the pyrimidine ring of cytosine nucleotide (5-methylcytosine, $5 \mathrm{mC})$ mediated by DNA methyltransferase enzymes, 
assisting the cells with an epigenetic signaling tool to modulate the expression of the implicated genes at their convenience. Usually, the altered cytosine residues are immediately adjacent to a guanine nucleotide (CpG site) (Kundu and Rao, 1999), forming the denominated CpG islands in most human gene promoters (Saxonov et al., 2006). Recently, three derivatives of $5 \mathrm{mC}$ have been discovered in the DNA demethylation pathway: 5-hydroxymethylcytosine $(5 \mathrm{hmC}), 5$-formylcytosine $(5 \mathrm{fC})$ and 5 -carboxylcytosine $(5 \mathrm{caC})$ (Ito et al., 2011; Tahiliani et al., 2009), which are believed to confer unique transcriptional potential to genes (Kellinger et al., 2012; Li and Liu, 2011).

Tumor cells are characterized by presenting a different methylome in comparison to normal cells and the change in the methylome does not occur randomly but it is unique for each cancer type (Marsit et al., 2006). As a consequence, aberrant cytosine methylation has been associated with the development of several cancers (Fang et al., 2011; Hughes et al., 2012; Suzuki et al., 2006; Villanueva et al., 2015). Moreover, DNA methylation profiling has proven to give a consistent diagnosis of the primary tumor in cases of cancer of unknown primary site (Moran et al., 2016). Quantitative analysis of these epigenetic marks is now considered for clinical applications, since it can be useful in the early diagnosis and also in the monitoring of tumor progression and response to treatment.

Current research for DNA methylation routine diagnostics focuses on the development of efficient devices combining an accurate quantification with high sensitivity and specificity, minimizing both the resources and the time employed. It will allow for faster, cost-effective and more reliable diagnostics, promoting the development of individualized therapies and competent follow-up treatments, positively impacting on the well being of the patient. In this regard, label-free optical biosensors have been highlighted as compelling candidates due to their outstanding potential which enables a fast, real-time analysis combined with high sensitivity, selectivity and simple-to-operate procedures (Carrascosa et al., 2016; Fan et al., 2008). Several label-free optical biosensor methodologies have been proposed for the detection of methylcytosine residues in the genome (Nazmul Islam et al., 2016; Syedmoradi et al., 2016). Most of them rely on the conversion of non-methylated cytosine residues into uracil residues by DNA bisulfite treatment and subsequent amplification of the converted sequence by polymerase chain reaction (PCR) 
(Carrascosa et al., 2014; Shiddiky et al., 2015; Shin et al., 2013). However, these steps make the detection time-consuming and susceptible to error, concluding in misleading analysis that might hinder a proper diagnosis. Other approaches that avoid the sample manipulation have been proposed, such as the recognition of methylated DNA through methyl-CpG binding domain (MBD) proteins in a two-step analysis (Pan et al., 2010; Yu et al., n.d.). These strategies avoid the use of bisulfite conversion, but the need for designing methylated probes hampers the standardization of the process. Another interesting approach avoiding pre-manipulation of the sample consists of a sequence specific immunoassay for specific DNA methylation assessment by microfluidic-based SPR detection (Kurita et al., 2015). However, in this protocol a pre-heating step is necessary for duplex hybridization, which may compromise the efficiency of the detection.

A solution to skip the laborious steps prior detection is the analysis of the methylation status directly from double-stranded DNA (ds-DNA) fragments. This approach will exclude possible errors introduced during PCR amplification or bisulfite conversion by dealing directly with the biological material of interest. It would also prevent from loss in sample recovery after pre-heating steps. In this regard, an interesting approach based on poly-purine reverse-Hoogsteen (PPRH) oligonucleotides was described by Coma et al. (Coma et al., 2005) PPRH oligonucleotides are DNA molecules composed of two mirrorsymmetrical polypurine stretches linked by a polythymidine loop. They can fold into reverse-Hoogsteen hairpins and bind to their polypyrimidine target sequence by Watson-Crick bonds forming a threestranded (triplex) conformation. More interestingly, PPRH oligonucleotides are capable of interacting with ds-DNA by targeting pyrimidine tracks located in the desired sequences and producing a strand displacement of the complementary polypurine strand. Due to the ability of forming triplex structures with ds-DNA, this approach has been exploited for gene therapy either by knockdown gene expression (de Almagro et al., 2011) or correction of point mutations (Noé et al., 2017; Solé et al., 2016, 2014).

We present here an optical biosensor methodology based on PPRH probes capable of capturing in a straightforward manner specific ds-DNA fragments by triple helix formation. For the demonstration, we have employed a portable custom-made SPR biosensor (Huertas et al., 2016a). Our biosensor methodolo- 
gy has been specifically designed for the analysis of DNA-methylation marks presented in a specific fragment of the promoter region of $P A X-5$ gene. $P A X-5$ gene plays an important role in cell differentiation and embryologic development and shows aberrant hypermethylation in tumor cell lines as well as in primary tumors of breast (Moelans et al., 2011) and lung cancer (Diaz-Lagares et al., 2016). Therefore, the assessment of the methylation level of such promoter gene region by a label-free optical biosensor is of great interest for the early detection, diagnosis, prognosis, therapeutic stratification and post-therapeutic monitoring of these cancers.

\section{MATERIALS AND METHODS}

Chemical reagents, buffers and DNA sequences. The list of reagents, buffers and DNA sequences used in this work and procedure of preparation of DNA probes are provided in the Suplementary material.

SPR biosensor. We employed a portable custom-made SPR sensor as previously described (Huertas et al., 2016a). The SPR biosensor platform is based on Kretschmann configuration and monitors refractive index (RI) changes in real time. A p-polarized light of $670 \mathrm{~nm}$ from a laser source is divided in two identical beams focused on the crystal-backside of the gold sensor chip (glass surface coated with $2 \mathrm{~nm}$ of chromium and $45 \mathrm{~nm}$ of gold, $10 \times 10 \times 0.3 \mathrm{~mm}^{3}$, SSens, Enschede - The Netherlands). Measurements are performed at a fixed angle of incidence. Variations of the RI are detected due to the biointeraction events occurring at the sensor surface as changes in the reflected light intensity by a multielement photodiode. The flow system consists of two flow cells (300 nL each) for independent analysis. The device incorporates all optics, electronics and fluidics components necessary to operate autonomously. Sensograms reproduce the binding event by monitoring the increase (or decrease in case of unbinding events) of the intensity of the reflected light ( $\Delta$ Reflectivity $(\%), \Delta \mathrm{R}(\%))$ vs. time (seconds, s). This change of the intensity of the reflected light is directly related to changes in the RI of the dielectric medium caused by mass changes on the metallic surface.

DNA probes immobilization. Prior to probe immobilization, gold sensor chips were cleaned by consecutive sonication cycles $(1 \mathrm{~min})$ with solvents of increasing polarity (i.e. acetone, ethanol and $\mathrm{dH}_{2} \mathrm{O}$ ) 
previously heated up to their boiling point. Then, substrates are dried under nitrogen flux and placed in an $\mathrm{UV} / \mathrm{O}_{3}$ generator (BioForce Nanosciences, USA) for $20 \mathrm{~min}$. After that, gold sensor chips were subsequently rinsed with ethanol and water and dried under nitrogen flux. The gold sensor chip is then placed into the SPR device.

Formation of self-assembled monolayers (SAMs) of DNA-probe/horizontal spacer $(1 \mu \mathrm{M})$ was carried out in-situ on the gold sensor chip by flowing $250 \mu \mathrm{L}$ of the mix in PB buffer solution at a $12 \mu \mathrm{L} / \mathrm{min}$ rate. Different horizontal spacer molecules (MCH and SH-PEG- $\mathrm{CH}_{3} 2000 \mathrm{MW}$ ) were employed depending on the experiment carried out.

DNA hybridization. Single-stranded and double-stranded DNA detections were performed by injection of $250 \mu \mathrm{L}$ of the samples into the SPR biosensor at $15 \mu \mathrm{L} / \mathrm{min}$ rate. The samples were dissolved in $5 \mathrm{x}$ saline sodium citrate (SSC) buffer with different percentages of formamide $(0,10,15,20,25$ and 30\%). Finally, target-probe interactions were disrupted by employing $20 \mathrm{mM} \mathrm{NaOH}$. Double-stranded DNA fragments were obtained by incubating both strands (sense (+) and -antisense (-)) of PAX-5 fragment at 95 ${ }^{\circ} \mathrm{C}$ for 5 min. Regarding the possibility of finding free $P A X-5+$ strands in the tested samples (i.e. not forming ds-DNA), an excess of $P A X-5$ - was employed for the incubation (1:3 molar ratio) to completely reduce the number of free $P A X-5+$ strands. After that, we cooled down the sequences at room temperature for $1 \mathrm{~h}$. Once generated the ds-PAX-5 fragments, we injected the samples in the SPR biosensor.

5-Methylcytosine quantification. Anti-5mC and anti-5hmC antibodies (GeneTex, Inc., USA) were diluted $2 \mu \mathrm{g} / \mathrm{mL}$ concentration in $0.5 \mathrm{x}$ SSC-0.1\% Tween 20 for 5-methyl-cytosine residue recognition in differently methylated DNA. $250 \mu \mathrm{L}$ of the samples containing the antibodies were injected into the SPR biosensor at $15 \mu \mathrm{L} / \mathrm{min}$ rate.

Data analysis. The sensor data were analysed and processed using Origin 8.0 software (OriginLab Northampton, MA). Bioanalytical chemistry analysis was performed by Graphpad Prism (Graphpad Software, Inc., California, USA). The experimental detection limit (LOD) was defined as the target concentration giving a $\Delta \mathrm{R}(\%)$ in the hybridization signal at least three times higher than the one from the 
standard deviation of the DNA control signal. The coefficients of variation were obtained as the ratio of the standard deviation of the mean, expressed in percentages (\% CV).

\section{RESULTS AND DISCUSSION}

DNA methylation biosensor. The SPR biosensor employed in this work is integrated in a reduced platform $\left(30 \times 30 \times 14 \mathrm{~cm}^{3}\right)$ and can be employed as a portable and user-friendly device (Huertas et al., 2016a). The gold sensor surface where the biomolecular interaction takes place is in contact with two identical flow cells of $300 \mathrm{~nL}$ volume each one. The laser beam is divided in two identical intensity beams using a light splitter to enable the simultaneous evaluation in the two independent flow cells. Biological events occurring at the sensor surface are detected as changes in the reflected light intensity ( $\Delta$ Reflectivity $(\%)$, $\Delta \mathrm{R}(\%))$ by a photodiode. This part of the set-up is mounted on a rotatory platform in order to select the optimum incidence angle that maximizes the changes of reflected light intensity. The platform also incorporates all electronics and fluidics components necessary to operate autonomously. The fluidic system consists of a peristaltic pump, which keeps a continuous flow and a set of tubing and valves for the sample handling and injection to the flow cuvette. 


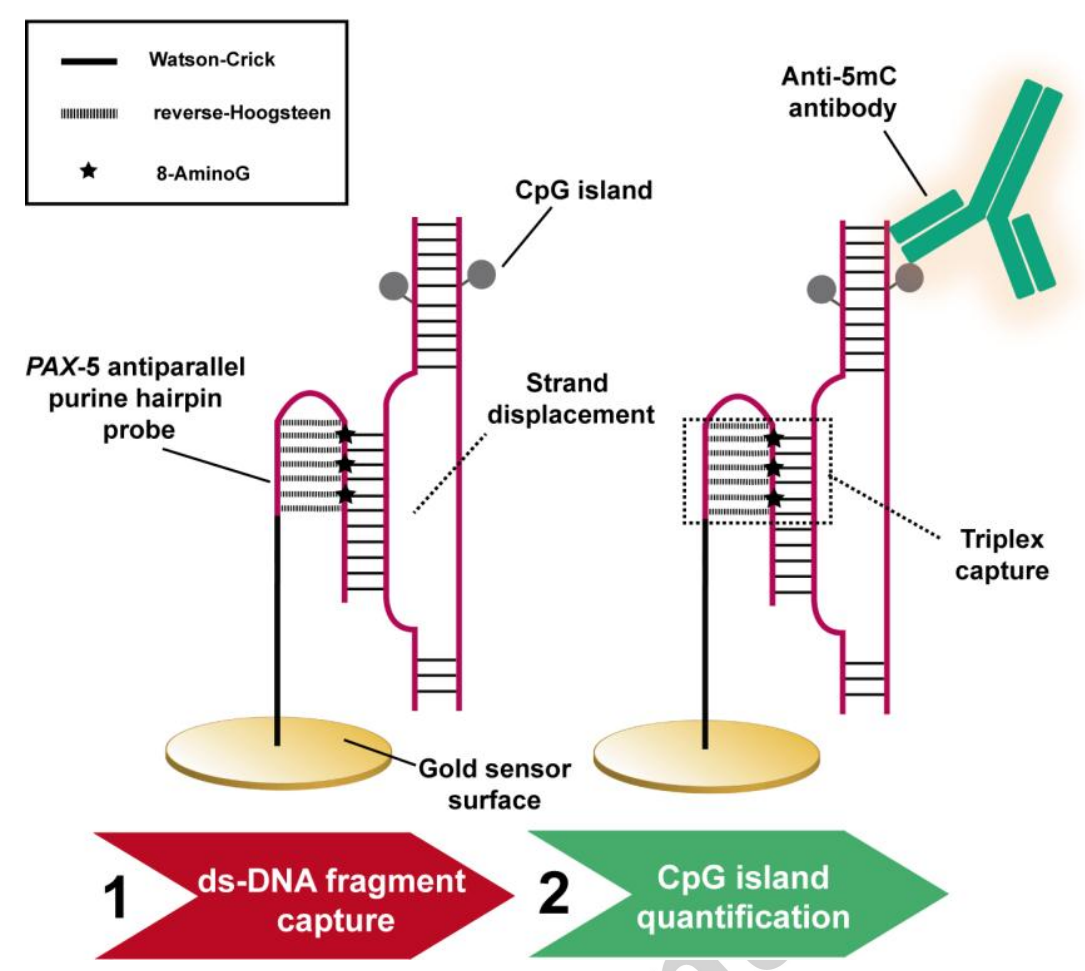

Figure 1. Scheme of the DNA methylation biosensor methodology. The methodology follows a two-step molecular recognition event: (1) Capture of biological ds-DNA fragments obtained after restriction enzyme treatment by the PPRH probe and subsequent (2) quantification of $5 \mathrm{mCpG}$ islands by specific anti-5mC antibody.

The scheme of the employed methodology is shown in Figure 1. The biosensor methodology is designed for capturing specific ds-DNA fragments generated by restriction enzymes. We have designed a PPRH probe consisting of two antiparallel poly-purine domains connected by a tetra-thymidine loop (Table S1). The PPRH probe was designed to capture the fragment of interest by nucleotide complementarity. Therefore, we selected a 14-homopurine sequence that hybridize to a 14-homopyrimidine sequence located in one DNA strand of the $P A X-5$ gene promoter region of interest by the formation of a triple helix, inducing the displacement of the other strand (Figure S1). During this strand displacement, the homopurine portion is extended in order to hybridize the target sequence by Watson-Crick interactions and the inverted homopurine portion of the clamp forms a triplex helix by reverse-Hoogsteen hydrogen bonding to the newly formed duplex. The designed probe introduces an innovative structural feature compared to 
the one previously described (Coma et al., 2005) by modifying the tail-clamp with three 8-amino-2'deoxyguanosine, which has been demonstrated to stabilize the hybridization, improving the biosensor performance (Aviñó et al., 2016). In addition, a thiol (SH-) group has been added to the 5' edge of the hairpin sequence in order to provide a linker for the biofunctionalization of the sensor surface. A sequence consisting of a poly-thymidine sequence $-(\mathrm{T})_{10^{-}}$is also placed between the functional group and the hairpin, acting as a vertical spacer, enhancing the target accessibility to the immobilized PPRH probes (Carrascosa et al., 2012). Thermal denaturation studies demonstrate the stronger affinity of the polypurine hairpin $\left(83.2{ }^{\circ} \mathrm{C}\right)$ compared with duplex-forming sequences for the $P A X-5$ polypyrimidine target $\left(76{ }^{\circ} \mathrm{C}\right)$ by a more than 7 degrees (Table S2). Furthermore, CD spectra of duplex and triplex solutions were clearly different providing experimental proofs for the formation of an antiparallel triplex (Figure S2). The sum of the CD spectra indicated the formation of a new structure (antiparallel triplex) that is the responsible of the increased stability.

In order to determine the cytosine DNA methylation status we employed an anti-5-methylcytosine (anti$5 \mathrm{mC}$ ) antibody. This antibody has been previously reported to recognize specifically cytosine residues from a DNA sequence modified with methyl groups (Kurita et al., 2015). The immunochemical recognition is performed once captured the ds-DNA fragment of interest, which produces a sensor signal proportional to the quantity of methyl groups, allowing the determination of the methylation status of the specific promoter region.

The probe accessibility was assessed by testing the influence of the introduction of horizontal spacers during the SAM formation, which promotes the orientation of the probes (Satjapipat et al., 2001), reduces their density and improves the resistance to non-specific adsorptions of nucleic acids to the surface (Lee et al., 2006). We compared the biosensor performance employing two different horizontal spacers: (i) 6mercapto-1-hexanol (MCH) spacer, a commonly used backfiller, and (ii) thiolated polyethyleneglycol (SH-PEG-ME, $2000 \mathrm{MW}$ ) polymer, which has shown to minimize the non-desired adsorptions to the sensor surface (Cerruti et al., 2008). Poly(ethylene glycol) (PEG) SAMs are commonly used to modify $\mathrm{Au}$ substrates for both, as a horizontal spacer and to prevent nonspecific adsorptions. Therefore, PEG 
SAMs in Au surfaces have been characterized extensively with many different techniques for a long time and proven their capabilities for surface functionalization.

We performed several hybridization cycles over each SAM by flowing samples containing ss-PAX-5 + sequence at $100 \mathrm{nM}$ concentration and monitoring the interactions in real time. The interaction between the PPRH probe and the target sequence was interrupted by flowing a mild concentration of sodium hydroxide $(\mathrm{NaOH})$, usually employed to reuse the biosensor surface in subsequent analyses and to test the reproducibility of the different monolayer configurations (Ulianas et al., 2014). As it can be observed in Figure S3, SAMs incorporating the horizontal spacers showed higher signal intensity than the homogeneous one $(P<0.0003$, one-way ANOVA test). However, the different horizontal spacers combined with our PPRH probe showed different stabilities. PEGylated monolayers provided with more reproducible signals after several cycles of hybridization/regeneration, presenting a Coefficient of Variation (CV) of 8\%, versus the MCH spacer, with a signal variation of $52 \%$ (Figure S4). In addition, the latter one showed a decrease in the signal intensity by $73 \%$ after each cycle, jeopardizing the reliability of the analysis.

Due to the higher consistence and reproducibility provided by the PEGylated surface, we employed this monolayer for further experiments. In order to improve the hybridization efficiency between the target and our PPRH probe, we investigated the effect of employing formamide (FA) during the detection. Solutions containing an adequate percentage of FA are known to reduce the presence of secondary structures and increase the specificity by lowering the melting temperature of oligonucleotides (Fuchs et al., 2010). Thus, one can obtain the best hybridization efficiency by a simple adjustment of the FA content without the need for a temperature controller. It also reduces the likelihood of interactions from non-fully complementary sequences, improving both the selectivity and the specificity with a reduction of potential biases from non-specific interactions.

We tested different concentrations of FA from zero to $30 \%$ in the 5x SSC hybridization buffer for the hybridization of ss-PAX-5 + at $30 \mathrm{nM}$ concentration. As can be observed in Figure $2 \mathrm{~A}$, the addition of $20 \%$ FA to the hybridization buffer helped to increase the signal by $221 \%(P<0.0001)$. On the contrary, higher percentages of FA largely reduced the sensor signal by decreasing the hybridization efficiency of 
the target with the PPRH probe, which could be due to a destabilization of the hybridization process by the highly stringent conditions employed.

Once improved the accessibility and the hybridization efficiency, we assessed the interaction between the fragment of interest and the probe. Optical biosensors monitor changes in the refractive index taking place in the sensor area generated by the interaction of any biomolecule. However, some of these interactions can be unspecific, either by cross-hybridization of other molecules or by non-specific adsorptions of molecules to the surface, giving up to false positive signals. Therefore, for the study of DNA interaction, it is essential to employ control sequences to prove the specificity of the signal given by the biosensor. To that purpose, we monitored the SPR response after flowing over the sensor surface two control sequences at $50 \mathrm{nM}$ concentration: (i) ss-PAX-5 - sequence, and (ii) a 100-nt control sequence. As can be appreciated in Figure 2B, the signals from both sequences dropped to the baseline level after their interaction with the monolayer, indicating that no-hybridization or non-specific adsorptions took place. Such results evidenced the specificity of the PPRH probe only to ss-PAX-5 + sequence in contrast with the negligible signal of the hairpin with the complementary strand ss-PAX-5 -.

After finding the most suitable detection conditions and having demonstrated the specificity of the probe for ss- $P A X-5+$ in contrast with ss-PAX-5 -, a calibration curve was obtained by employing different concentrations of ss-PAX-5 + (from 2 to $50 \mathrm{nM}$ ) (Figure 2C). The theoretic limit of detection (LOD), defined as the target concentration giving a $\Delta \mathrm{R}(\%)$ in the hybridization signal at least three times higher than the one from the standard deviation of the DNA control signal, was $115 \mathrm{pM}\left(\mathrm{R}^{2}=0.98\right)$ which is within the appropriate range of detection in SPR biosensors (Šípová and Homola, 2013). The signal variation $(8 \%)$ is found below the maximum variability recommended for analytical methods $(\sim 15 \%)$ (Wood, 1999) up to 35 hybridization/regeneration cycles, after which the monolayer started losing its hybridization efficiency (Figure 2D), which demonstrated the capability of our methodology to be reused for several analyses. 

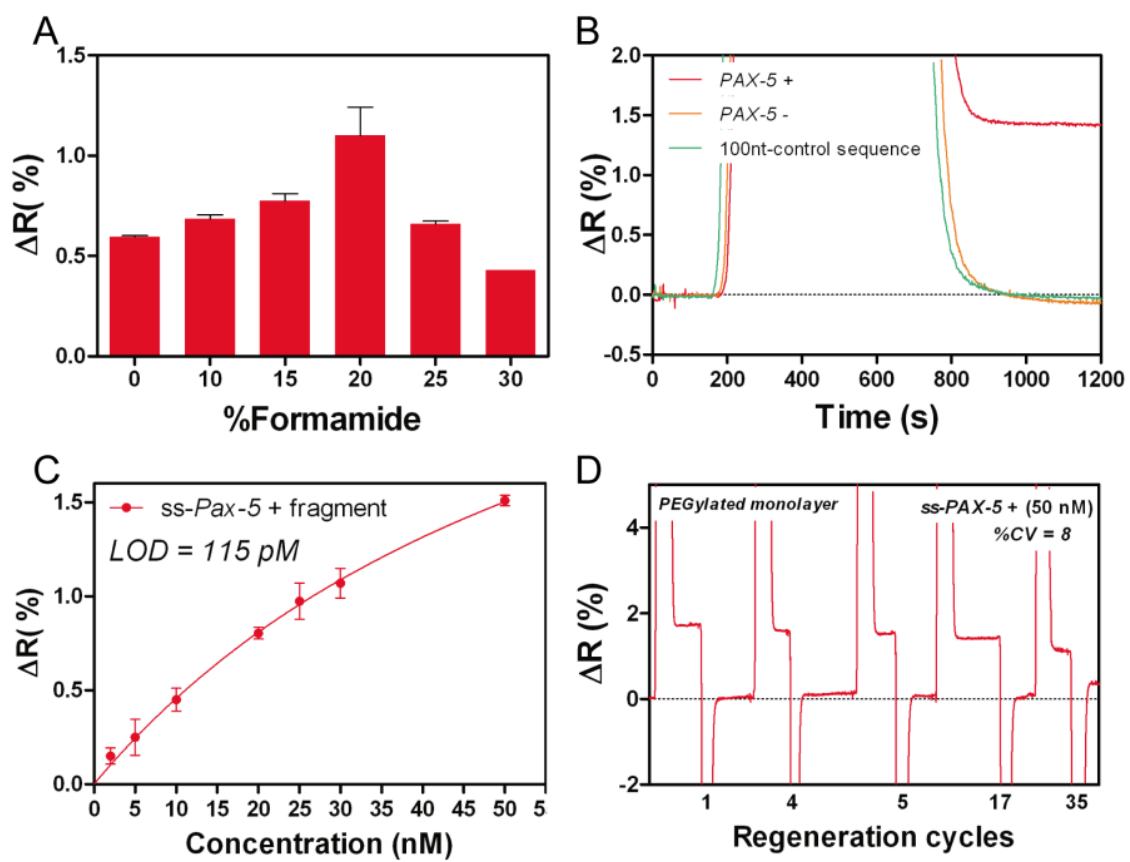

Figure 2. Optimization of ss- $P A X-5+$ fragment detection with the PEGylated monolayer. (A) Influence in the hybridization efficiency by the addition of different percentages of FA (from 0 to $30 \% \mathrm{v} / \mathrm{v}$ ) to the hybridization buffer. All p-values indicated significant variation between the measurements $(P<0.0001$, one-way ANOVA test). (B) PPRH probe hybridization assessment by real-time monitoring of the response to different DNA sequences (ss-PAX$5+$, ss-PAX-5 - and a 100-nt control sequence). (C) SPR calibration curve for ss-PAX-5 + fragment. Solid line (red) corresponds to the non-linear fit of the calibration curve. All data show mean \pm SD of triplicate measurements. (D) Detection cycles after consecutive interactions of ss-PAX-5 + (50 nM) with the specific PPRH probe and subsequent surface regenerations with $\mathrm{NaOH} 20 \mathrm{mM}$.

Anti-5-methylcytosine antibody-capture assessment. The specificity of the anti-5mC antibody for 5methylcytosine modifications was assessed. For that purpose, we monitored the DNA hybridization and the antibody recognition sequentially in real time. We employed two different ss- $P A X-5+$ sequences, one with no methyl groups (non-methylated) and other one modified with 4 methyl groups $(4 \mathrm{x} 5 \mathrm{mC})$ (Table $\mathrm{S} 1)$. The DNA sequences were diluted in 5x SSC at 20-nM concentration. Once hybridized, we flowed a solution containing $2 \mu \mathrm{g} / \mathrm{mL}$ of anti-5mC antibody. As can be observed in the sensogram (Figure 3A), both targets produced the same increment in the reflectivity, showing the same hybridization efficiency 
despite of the 5-methyl-cytosine content. However, when evaluating the antibody capture, only the sequence containing the $5 \mathrm{mCs}$ gave rise to a positive signal $(\Delta \mathrm{R}(\%)=0.7)$. These results indicated that the antibody capture was specific for the methylated sequences and no interaction occurs when the hybridized fragment is not methylated. Also, in order to check the cross-reactivity of methyl-groups with other antibodies, we tested the interaction of DNA methylated sequences with anti 5-hydroxymethylcytosine $(5 \mathrm{hmC})$ antibody, an antibody that specifically recognizes $5 \mathrm{hmC}$ residues in DNA sequences (Ladopoulos et al., 2013). For that purpose, 4 times methylated $P A X-5+$ fragment and subsequent antibody interaction were analyzed at the same conditions. As it can be observed in Figure S5, no signal increment was perceived when employing the anti-5hmC control antibody, suggesting that no cross-reaction took place.

Then, we assessed the quantitative analysis of methylcytosines by employing 20-nM concentration of ss-PAX-5 + fragments showing different methylation profiles: non-methylated, $1 \mathrm{x} 5 \mathrm{mC}$ and 4x 5mC (Table S1). The different sequences were flowed to the sensor surface at the optimized conditions and the quantification of 5-methylcytosines was performed by subsequent anti-5mC antibody recognition. As it can be observed in Figure3B, the antibody signal increased significantly $(P<0.0014)$ with increasing number of methyl groups in the captured DNA sequence. While fragments with no methyl-modifications gave rise to negligible sensor responses, the presence of methyl groups in the sequence produced positive sensor signals. Moreover, the sensor response to the anti-5mC antibody increased by $75 \%$ in fragments containing four methyl groups compared with the ones with only one methyl-modification. This increase corresponded with a 4 times sensor response, which highly correlates with the different DNA methylation status between the two sequences. These results corroborated the capacity of the biosensor for the correct quantification of DNA-methyl groups. In addition, this immune-recognition step allows bisulfiteconversion free detections, increasing the speed of the analysis and preventing possible bias introduction by a wrong conversion. 
A

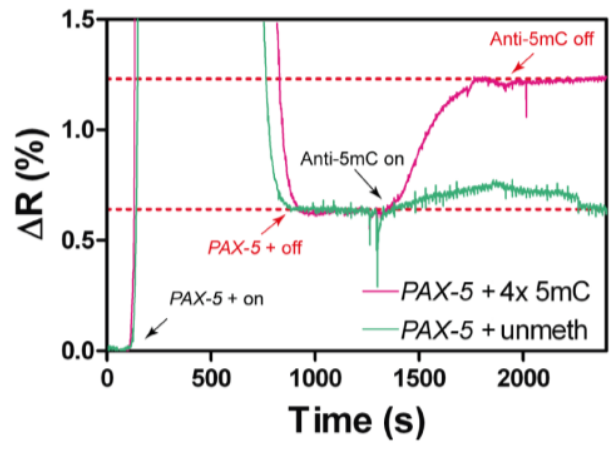

B

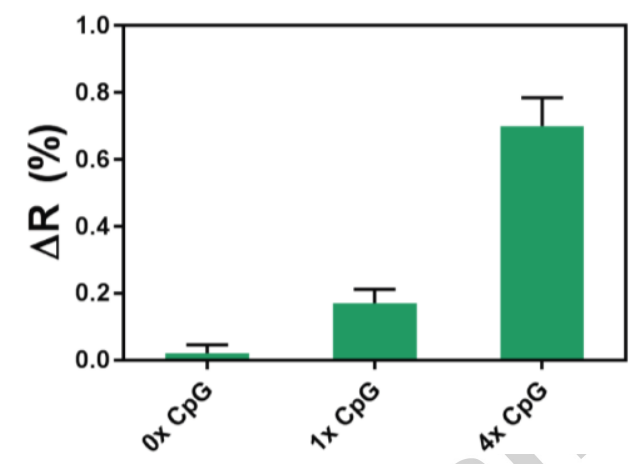

Figure 3. Anti-5-mC antibody specificity assessment. (A) SPR sensogram showing the antibody interaction with two differently methylated ss-PAX-5 + sequences: unmmethylated (green) and four 5-methylcytosines (pink). (B) Interaction of anti-5-methyl-CpG antibody with three different methylation profiles $(0 \mathrm{x}, 1 \mathrm{x}$ and $4 \mathrm{x}$ CpGs). All pvalues indicated significant variation between the signals from the different $\mathrm{CpG}$ content $(P<0.0014$, one-way ANOVA test). All data show mean $\pm \mathrm{SD}$ of triplicate measurements.

Double-stranded $P A X-5$ gene fragment capture. Once optimized the biofunctionalization and hybridization conditions for ss-PAX-5 + detection, we tested the capability of our methodology for capturing dsDNA fragments and determining their methylation status. Thus, we compared the performance of a standard duplex-forming probe with the PPRH probe, both complementary to the same sequence contained in the ss-PAX-5 + strand. Taking advantage of the full specificity of ss-PAX-5 + to our hairpin in contrast with the null specificity showed by ss-PAX-5 - strand, we analyzed the methylation status of the latter one, which will only be possible if ds-DNA PAX-5 fragment is captured. We employed 2-times methylated version of ss- $P A X-5$ - strand. Therefore, only if ds-PAX-5 fragments hybridized with our probes by a 
strand displacement process, the sensor would produce a positive signal in the second step of antibody capture. On the contrary, if no interaction took place, no sensor response would be appreciated. As in previous assays, the ds-PAX-5 fragments were diluted in 5x SSC- $20 \%$ FA at $20 \mathrm{nM}$ concentration and flowed over the sensor surface.

The sensor response for the ds-PAX-5 fragment capture and subsequent interaction with the anti-5mC antibody for both probes are shown in Figure 4. As can be appreciated, while the sensor signal from the PPRH probe (pink) showed an increase in the baseline level $(\Delta \mathrm{R}(\%)=0.27)$, the signal coming from the duplex-forming probe (green) recovered the initial baseline level after ds-PAX-5 fragment injection, which indicates that no interaction took place. These results demonstrated the PPRH probe capabilities for capturing ds-DNA fragments by strand-displacement in contrast with the inability of standard duplexforming probes to hybridize with the double-stranded fragment. In addition, during the antibody capture, only the triplex-forming probe led to the specific detection of the antibody, which corroborated the proper capture of ds-PAX-5 by this probe and the specific antibody recognition of the methyl group in the $P A X-5$ - strand. Although the hybridization efficiency of the ds-DNA fragments at $20 \mathrm{nM}$ concentration was reduced by $50 \%(\Delta \mathrm{R}=0.35)$ in comparison with the single-stranded $P A X-5+$ fragment $(\Delta \mathrm{R}=0.7)$, probably due to the different kinetics of the ds-DNA fragment hybridization, the PPRH probe was able to capture ds-DNA fragments directly from the sample. Thus, our methodology can be easily implemented without the need for laborious pre-manipulation steps such as bisulfite conversion or PCR amplification. 


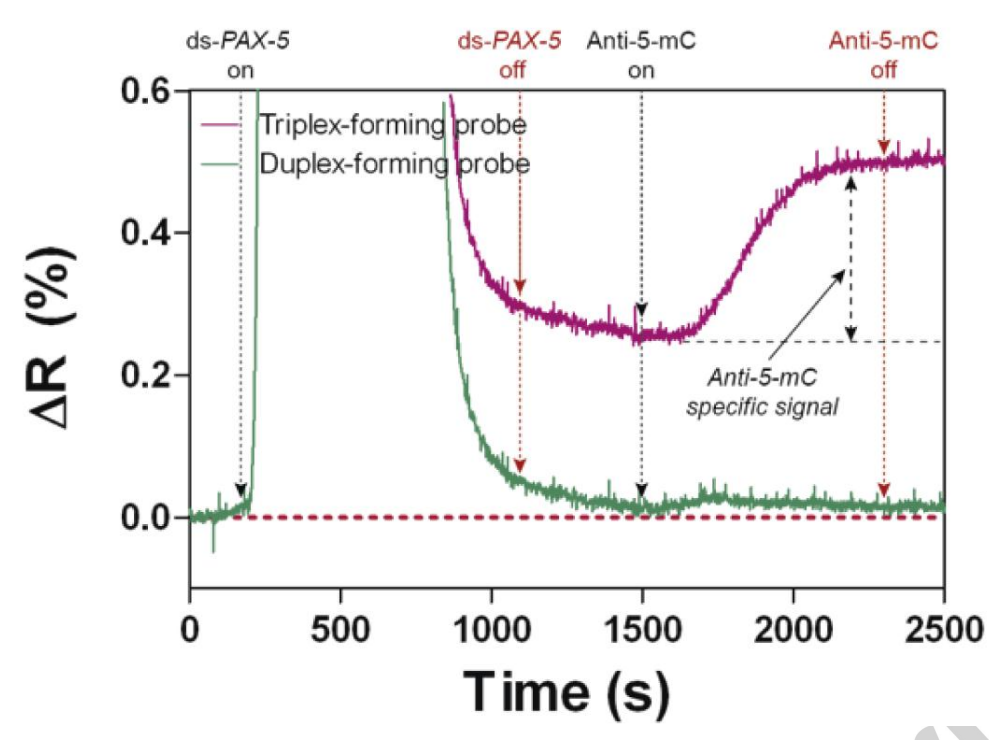

Figure 4. Sensogram of the ds-DNA recognition assessment. ds- $P A X-5$ fragment interaction with both, PPRH probe (pink) and conventional duplex probe (green), and subsequent quantification of $5 \mathrm{mC}$ by the specific anti-5mC antibody.

\section{CONCLUSIONS}

The determination of the DNA methylation status in patients is of paramount relevance in order to determine the cancer of primary origin at very early stages. Moreover, monitoring DNA methylation profiles before and after cancer treatment may yield evidence concerning the efficiency of the applied therapy or to detect disease recurrence. Aware of the necessity of faster and more efficient diagnostic tools, we have developed an innovative methodology for DNA methylation analysis through a label-free optical biosensor. Based on the demonstrated capabilities of PPRH oligonucleotides for inducing strand displacement in ds-DNA, we designed an antiparallel hairpin probe capable of directly hybridizing ds-DNA fragments. The employment of our PPRH-probe skips previous DNA strand de-hybridization or PCR amplification, avoiding the introduction of potential errors since we are dealing directly with the biological material of interest. We increased the strength of the capture by modifying the hairpin probe sequence with 8 -aminoG modifications, which has been previously demonstrated to enhance the stabilization of the triple helix structure (Aviñó et al., 2003; Robles et al., 2002; Soliva et al., 2000). 
This biosensor methodology has proven to be useful for the complete characterization of the methylation status in DNA fragments. No cross-reactivity was observed when employing a non-specific antibody. Therefore, it can be also employed for the screening of other DNA-modifications such as 5hydroxymethylcytosines, 5-formylcytosine and 5-carboxylcytosine. Alterations in the percentage of these different epigenetic modifications have been reported in cancer by enzyme-based immunoassay (Chowdhury et al., 2014). Thus, our methodology could enable the detection of each of these epigenetic marks independently by employing the specific antibody required for each one. This biosensor strategy provides a more reliable and fast analysis than conventional techniques, which is essential for the complete screening of these epigenetic marks in cancer patients whose epigenetic status has been altered. Table S3 compares the performances of the existing DNA-methylation optical biosensors. These results also place our methodology in the front line for the development of biosensor devices that may require the direct capture of ds-DNA fragments, such as circulating cell-free DNA, and the presence of bacteria or viruses in the host organism. In order to improve the accessibility of the ds-DNA, which probably endure higher steric hindrance repulsions during the interaction due to the double-stranded conformation fragments, a re-establishment of the biofunctionalization conditions by employing different PPRH probe:SHPEG ratios may be required. Further experiments should be performed with the established conditions for the evaluation of real samples from cancer patients and healthy individuals and properly validated with the corresponding conventional techniques in order to demonstrate de feasibility of our methodology. Also, to achieve a competent sensitivity for the evaluation of DNA methylation in genomic DNA, our future research is focused on the transference of the methodology to a highly sophisticated biosensor platform based on bimodal waveguide (BiMW) interferometers. This nanophotonic biosensor has demonstrated a high sensitivity, reaching limits of detections up to the low-attomolar level in a direct detection (Huertas et al., 2016b), avoiding any type of amplification process, such as PCR amplification.

\section{ACKNOWLEDGMENT}


This work was partially supported by grants of the Spanish Ministry of Economy (MINECO) (CTQ201020541 and CTQ2014-52588-R), CERCA Programme/Generalitat de Catalunya (2009/SGR/208, 2014/SGR/624), and CIBER-BBN. CIBER-BBN is an initiative funded by the VI National R\&D\&I Plan 2008-2011, Iniciativa Ingenio 2010, Consolider Program, and CIBER. Actions and financed by the Instituto de Salud Carlos III with assistance from the European Regional Development Fund. ICN2 is supported by the Severo Ochoa program from Spanish MINECO (Grant No. SEV-2013-0295). J.S is a Miguel Servet researcher (MS13/00055).

\section{Author Contributions}

C.S.H., A.A., J.S., R.E., M.E. and L.M.L. designed the study; C.S.H., A.A., C.K. and A.P. performed the experiments; A.A. and R.E. designed and synthesized the PPRH probes; C.S.H. and L.M.L. analyzed the data; C.S.H. and L.M.L. wrote the main manuscript. C.S.H. prepared the figures. All authors reviewed the manuscript.

\section{Conflict of intestest}

The authors declare that they have no conflict of interest.

\section{REFERENCES}

Aviñó, A., Cubero, E., González, C., Ramon Eritja, A., Orozco, M., 2003. Antiparallel Triple Helices. Structural Characteristics and Stabilization by 8-Amino Derivatives. https://doi.org/10.1021/JA035039T

Aviñó, A., Huertas, C.S., Lechuga, L.M., Eritja, R., 2016. Sensitive and label-free detection of miRNA145 by triplex formation. Anal. Bioanal. Chem. 408, 885-893.

Carrascosa, L.G., Gómez-Montes, S., Aviñó, A., Nadal, A., Pla, M., Eritja, R., Lechuga, L.M., 2012. Sensitive and label-free biosensing of RNA with predicted secondary structures by a triplex affinity capture method. Nucleic Acids Res. 40. https://doi.org/10.1093/nar/gkr1304 
Carrascosa, L.G., Huertas, C.S., Lechuga, L.M., 2016. Prospects of optical biosensors for emerging labelfree RNA analysis. TrAC - Trends Anal. Chem.

Carrascosa, L.G., Sina, A.A.I., Palanisamy, R., Sepulveda, B., Otte, M. a, Rauf, S., Shiddiky, M.J. a, Trau, M., 2014. Molecular inversion probe-based SPR biosensing for specific, label-free and realtime detection of regional DNA methylation. Chem. Commun. (Camb). 50, 3585-8. https://doi.org/10.1039/c3cc49607d

Cerruti, M., Fissolo, S., Carraro, C., Ricciardi, C., Majumdar, A., Maboudian, R., 2008. Poly(ethylene glycol) Monolayer Formation and Stability on Gold and Silicon Nitride Substrates. Langmuir 24, 10646-10653. https://doi.org/10.1021/la801357v

Chowdhury, B., Cho, I.-H., Hahn, N., Irudayaraj, J., 2014. Quantification of 5-methylcytosine, 5hydroxymethylcytosine and 5-carboxylcytosine from the blood of cancer patients by an enzymebased immunoassay. Anal. Chim. Acta 852, 212-7. https://doi.org/10.1016/j.aca.2014.09.020

Coma, S., Noé, V., Eritja, R., Ciudad, C.J., 2005. Strand displacement of double-stranded DNA by triplex-forming antiparallel purine-hairpins. Oligonucleotides $\quad 15, \quad 269-283$. https://doi.org/10.1089/oli.2005.15.269

de Almagro, M.C., Mencia, N., Noé, V., Ciudad, C.J., 2011. Coding Polypurine Hairpins Cause TargetInduced Cell Death in Breast Cancer Cells. Hum. Gene Ther. 22, 451-463. https://doi.org/10.1089/hum.2010.102

Diaz-Lagares, A., Mendez-Gonzalez, J., Hervas, D., Saigi, M., Pajares, M.J., Garcia, D., Crujerias, A.B., Pio, R., Montuenga, L.M., Zulueta, J., Nadal, E., Rosell, A., Esteller, M., Sandoval, J., 2016. A Novel Epigenetic Signature for Early Diagnosis in Lung Cancer. Clin. Cancer Res. 22.

Ellsworth, R.E., Blackburn, H.L., Shriver, C.D., Soon-Shiong, P., Ellsworth, D.L., 2017. Molecular heterogeneity in breast cancer: State of the science and implications for patient care. Semin. Cell Dev. Biol. 64, 65-72. https://doi.org/10.1016/j.semcdb.2016.08.025

Fan, X., White, I.M., Shopova, S.I., Zhu, H., Suter, J.D., Sun, Y., 2008. Sensitive optical biosensors for unlabeled targets: A review. Anal. Chim. Acta 620, 8-26. https://doi.org/10.1016/j.aca.2008.05.022 
Fang, F., Turcan, S., Rimner, A., Kaufman, A., Giri, D., Morris, L.G.T., Shen, R., Seshan, V., Mo, Q., Heguy, A., Baylin, S.B., Ahuja, N., Viale, A., Massague, J., Norton, L., Vahdat, L.T., Moynahan, M.E., Chan, T.A., 2011. Breast cancer methylomes establish an epigenomic foundation for metastasis. Sci. Transl. Med. 3, 75ra25. https://doi.org/10.1126/scitranslmed.3001875

Feinberg, A.P., Vogelstein, B., 1983. Hypomethylation distinguishes genes of some human cancers from their normal counterparts. Nature 301, 89-92. https://doi.org/10.1038/301089a0

Fuchs, J., Dell'Atti, D., Buhot, A., Calemczuk, R., Mascini, M., Livache, T., 2010. Effects of formamide on the thermal stability of DNA duplexes on biochips. Anal. Biochem. 397, 132-134. https://doi.org/10.1016/j.ab.2009.09.044

Heard, E., Martienssen, R.A., 2014. Transgenerational Epigenetic Inheritance: Myths and Mechanisms. Cell 157, 95-109. https://doi.org/10.1016/j.cell.2014.02.045

Huertas, C.S., Carrascosa, L.G., Bonnal, S., Valcárcel, J., Lechuga, L.M., 2016a. Quantitative evaluation of alternatively spliced mRNA isoforms by label-free real-time plasmonic sensing. Biosens. Bioelectron. 78, 118-125.

Huertas, C.S., Fariña, D., Lechuga, L.M., 2016b. Direct and Label-Free Quantification of Micro-RNA181a at Attomolar Level in Complex Media Using a Nanophotonic Biosensor. ACS Sensors acssensors.6b00162. https://doi.org/10.1021/acssensors.6b00162

Hughes, L.A.E., Khalid-de Bakker, C.A.J., Smits, K.M., van den Brandt, P.A., Jonkers, D., Ahuja, N., Herman, J.G., Weijenberg, M.P., van Engeland, M., 2012. The CpG island methylator phenotype in colorectal cancer: Progress and problems. Biochim. Biophys. Acta - Rev. Cancer 1825, 77-85. https://doi.org/10.1016/j.bbcan.2011.10.005

Ito, S., Shen, L., Dai, Q., Wu, S.C., Collins, L.B., Swenberg, J.A., He, C., Zhang, Y., 2011. Tet proteins can convert 5-methylcytosine to 5-formylcytosine and 5-carboxylcytosine. Science (80-. ). 333, 1300-3. https://doi.org/10.1126/science.1210597

Jankowska, A.M., Millward, C.L., Caldwell, C.W., 2015. The potential of DNA modifications as biomarkers and therapeutic targets in oncology. Expert Rev. Mol. Diagn. 15, 1325-1337. 
https://doi.org/10.1586/14737159.2015.1084229

Kellinger, M.W., Song, C.-X., Chong, J., Lu, X.-Y., He, C., Wang, D., 2012. 5-formylcytosine and 5carboxylcytosine reduce the rate and substrate specificity of RNA polymerase II transcription. Nat. Struct. Mol. Biol. 19, 831-833. https://doi.org/10.1038/nsmb.2346

Kundu, T.K., Rao, M.R., 1999. CpG islands in chromatin organization and gene expression. J. Biochem. $125,217-22$.

Kurita, R., Yanagisawa, H., Yoshioka, K., Niwa, O., 2015. On-Chip Sequence-Specific Immunochemical Epigenomic Analysis Utilizing Outward-Turned Cytosine in a DNA Bulge with Handheld Surface Plasmon Resonance Equipment. Anal. Chem. 87, 11581-11586. https://doi.org/10.1021/acs.analchem.5b03520

Ladopoulos, V., Hofemeister, H., Hoogenkamp, M., Riggs, A.D., Stewart, A.F., Bonifer, C., 2013. The histone methyltransferase KMT2B is required for RNA polymerase II association and protection from DNA methylation at the MagohB CpG island promoter. Mol. Cell. Biol. 33, 1383-93. https://doi.org/10.1128/MCB.01721-12

Lee, C.Y., Gong, P., Harbers, G.M., Grainger, D.W., Castner, D.G., Gamble, L.J., 2006. Surface coverage and structure of mixed DNA/Alkylthiol monolayers on gold: Characterization by XPS, NEXAFS, and fluorescence intensity measurements. Anal. Chem. 78, 3316-3325. https://doi.org/10.1021/ac052137j

Li, W., Liu, M., 2011. Distribution of 5-hydroxymethylcytosine in different human tissues. J. Nucleic Acids 2011, 870726. https://doi.org/10.4061/2011/870726

Marsit, C.J., Houseman, E.A., Christensen, B.C., Eddy, K., Bueno, R., Sugarbaker, D.J., Nelson, H.H., Karagas, M.R., Kelsey, K.T., 2006. Examination of a CpG Island Methylator Phenotype and Implications of Methylation Profiles in Solid Tumors. Cancer Res. 66, 10621-10629. https://doi.org/10.1158/0008-5472.CAN-06-1687

Moelans, C.B., Verschuur-Maes, A.H., van Diest, P.J., 2011. Frequent promoter hypermethylation of BRCA2, CDH13, MSH6, PAX5, PAX6 and WT1 in ductal carcinoma in situ and invasive breast 
cancer. J. Pathol. 225, 222-231. https://doi.org/10.1002/path.2930

Moran, S., Martínez-Cardús, A., Sayols, S., Musulén, E., Balañá, C., Estival-Gonzalez, A., Moutinho, C., Heyn, H., Diaz-Lagares, A., de Moura, M.C., Stella, G.M., Comoglio, P.M., Ruiz-Miró, M., MatiasGuiu, X., Pazo-Cid, R., Antón, A., Lopez-Lopez, R., Soler, G., Longo, F., Guerra, I., Fernandez, S., Assenov, Y., Plass, C., Morales, R., Carles, J., Bowtell, D., Mileshkin, L., Sia, D., Tothill, R., Tabernero, J., Llovet, J.M., Esteller, M., 2016. Epigenetic profiling to classify cancer of unknown primary: a multicentre, retrospective analysis. Lancet Oncol. 17, 1386-1395. https://doi.org/10.1016/S1470-2045(16)30297-2

Nazmul Islam, M., Yadav, S., Hakimul Haque, M., Munaz, A., Islam, F., Al Hossain, M.S., Gopalan, V., Lam, A.K., Nguyen, N.-T., Shiddiky, M.J.A., 2016. Optical biosensing strategies for DNA methylation analysis. Biosens. Bioelectron. https://doi.org/10.1016/j.bios.2016.10.034

Noé, V., Ciudad, C., Rodríguez, L., Villalobos, X., Félix, A., 2017. Polypurine Reverse Hoogsteen Hairpins as a Gene Silencing Tool for Cancer. Curr. Med. Chem. 24, 1-1. https://doi.org/10.2174/0929867324666170301114127

Pan, S., Xu, J., Shu, Y., Wang, F., Xia, W., Ding, Q., Xu, T., Zhao, C., Zhang, M., Huang, P., Lu, S., 2010. Double recognition of oligonucleotide and protein in the detection of DNA methylation with surface plasmon resonance biosensors. Biosens. Bioelectron. 26, 850-853. https://doi.org/10.1016/j.bios.2010.08.007

Robles, J., Grandas, A., Pedroso, E., Luque, F., Eritja, R., Orozco, M., 2002. Nucleic Acid Triple Helices: Stability Effects of Nucleobase Modifications. Curr. Org. Chem. 6, 1333-1368. https://doi.org/10.2174/1385272023373482

Sartore-Bianchi, A., Siena, S., Tonini, G., Bardelli, A., Santini, D., 2016. Overcoming dynamic molecular heterogeneity in metastatic colorectal cancer: Multikinase inhibition with regorafenib and the case of rechallenge with anti-EGFR. Cancer Treat. Rev. 51, 54-62. https://doi.org/10.1016/j.ctrv.2016.10.006

Satjapipat, M., Sanedrin, R., Zhou, F., 2001. Selective desorption of alkanethiols in mixed self-assembled 
monolayers for subsequent oligonucleotide attachment and DNA hybridization. Langmuir 17, 76377644. https://doi.org/10.1021/la010989i

Saxonov, S., Berg, P., Brutlag, D.L., 2006. A genome-wide analysis of CpG dinucleotides in the human genome distinguishes two distinct classes of promoters. Proc. Natl. Acad. Sci. U. S. A. 103, 14121417. https://doi.org/10.1073/pnas.0510310103

Shiddiky, M.J.A., Sina, A.A.I., Carrascosa, L.G., Palanisamy, R., Rauf, S., Trau, M., 2015. Methylsorb: A simple method for quantifying DNA methylation using DNA-gold affinity interactions, in: 8th International Conference on Electrical and Computer Engineering: Advancing Technology for a Better Tomorrow, ICECE 2014. pp. 17-20. https://doi.org/10.1109/ICECE.2014.7027002

Shin, Y., Perera, A.P., Kee, J.S., Song, J., Fang, Q., Lo, G.-Q., Park, M.K., 2013. Label-free methylation specific sensor based on silicon microring resonators for detection and quantification of DNA methylation biomarkers in bladder cancer. Sensors Actuators B Chem. 177, 404-411. https://doi.org/10.1016/j.snb.2012.11.058

Šípová, H., Homola, J., 2013. Surface plasmon resonance sensing of nucleic acids: A review. Anal. Chim. Acta. https://doi.org/10.1016/j.aca.2012.12.040

Solé, A., Ciudad, C.J., Chasin, L.A., Noé, V., 2016. Correction of point mutations at the endogenous locus of the dihydrofolate reductase gene using repair-PolyPurine Reverse Hoogsteen hairpins in mammalian cells. Biochem. Pharmacol. 110-111, 16-24. https://doi.org/10.1016/j.bcp.2016.04.002

Solé, A., Villalobos, X., Ciudad, C.J., Noé, V., 2014. Repair of single-point mutations by polypurine reverse Hoogsteen hairpins. Hum. Gene Ther. Methods 25, 288-302. https://doi.org/10.1089/hgtb.2014.049

Sölétormos, G., Duffy, M.J., Othman Abu Hassan, S., Verheijen, R.H.M., Tholander, B., Bast, R.C., Gaarenstroom, K.N., Sturgeon, C.M., Bonfrer, J.M., Petersen, P.H., Troonen, H., CarloTorre, G., Kanty Kulpa, J., Tuxen, M.K., Molina, R., Molina, R., 2016. Clinical Use of Cancer Biomarkers in Epithelial Ovarian Cancer: Updated Guidelines From the European Group on Tumor Markers. Int. J. Gynecol. Cancer 26, 43-51. https://doi.org/10.1097/IGC.0000000000000586 
Soliva, R., Güimil García, R., Blas, J.R., Eritja, R., Asensio, J.L., González, C., Luque, F.J., Orozco, M., 2000. DNA-triplex stabilizing properties of 8-aminoguanine. Nucleic Acids Res. 28, 4531-4539. https://doi.org/10.1093/nar/28.22.4531

Suzuki, M., Shigematsu, H., Iizasa, T., Hiroshima, K., Nakatani, Y., Minna, J.D., Gazdar, A.F., Fujisawa, T., 2006. Exclusive mutation in epidermal growth factor receptor gene, HER-2, and KRAS, and synchronous methylation of nonsmall cell lung cancer. Cancer 106, 2200-2207. https://doi.org/10.1002/cncr.21853

Syedmoradi, L., Esmaeili, F., Norton, M.L., 2016. Towards DNA methylation detection using biosensors. Analyst 141, 5922-5943. https://doi.org/10.1039/C6AN01649A

Tahiliani, M., Koh, K.P., Shen, Y., Pastor, W.A., Bandukwala, H., Brudno, Y., Agarwal, S., Iyer, L.M., Liu, D.R., Aravind, L., Rao, A., 2009. Conversion of 5-methylcytosine to 5-hydroxymethylcytosine in mammalian DNA by MLL partner TET1. Science (80-. ). 324, 930-5. https://doi.org/10.1126/science.1170116

Ulianas, A., Heng, L.Y., Ahmad, M., Lau, H.Y., Ishak, Z., Ling, T.L., 2014. A regenerable screen-printed DNA biosensor based on acrylic microsphere-gold nanoparticle composite for genetically modified soybean determination. Sensors Actuators, B Chem. 190, 694-701. https://doi.org/10.1016/j.snb.2013.09.040

Villanueva, A., Portela, A., Sayols, S., Battiston, C., Hoshida, Y., Méndez-González, J., Imbeaud, S., Letouzé, E., Hernandez-Gea, V., Cornella, H., Pinyol, R., Solé, M., Fuster, J., Zucman-Rossi, J., Mazzaferro, V., Esteller, M., Llovet, J.M., 2015. DNA methylation-based prognosis and epidrivers in hepatocellular carcinoma. Hepatology 61, 1945-1956. https://doi.org/10.1002/hep.27732

Wood, R., 1999. How to validate analytical methods. TrAC - Trends Anal. Chem. 18, 624-632. https://doi.org/10.1016/S0165-9936(99)00150-8

Yu, Y., Blair, S., Gillespie, D., Jensen, R., Myszka, D.G., Badran, A.H., Ghosh, I., Chagovetz, A., n.d. Direct DNA Methylation Profiling Using Methyl Binding Domain Proteins. https://doi.org/10.1021/ac1010316 


\section{GRAPHICAL ABSTRACT}
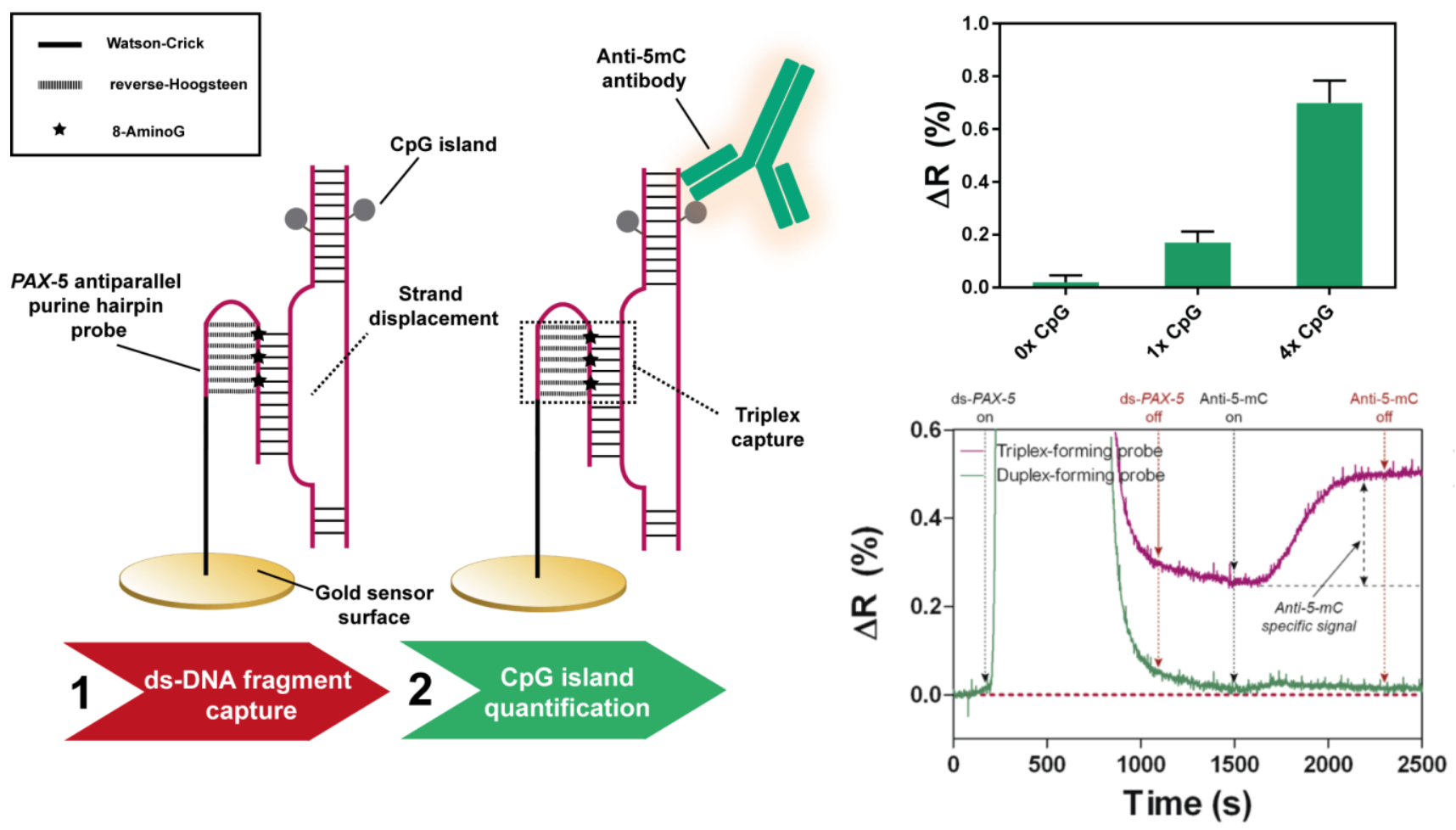


\section{Highlights:}

- Unique poly-purine hairpin probe that interacts directly with double-stranded DNA.

- We have demonstrated its potential as a powerful tool for label-free, real-time and direct DNA methylation analysis.

- Stronger affinity of the poly-purine hairpin over the duplex-forming sequences is demonstrated.

- Our biosensor is able to assess the methylation status by quantitative methylation-dependent responses. 